\title{
Circulating microRNAs as biomarkers for diffuse myocardial fibrosis in patients with hypertrophic cardiomyopathy
}

\author{
Lu Fang ${ }^{1 *}$, Andris H. Ellims ${ }^{1,2}$, Xiao-lei Moore ${ }^{1}$, David A. White ${ }^{1}$, Andrew J. Taylor ${ }^{1,2}$, Jaye Chin-Dusting ${ }^{1}$ \\ and Anthony M. Dart ${ }^{1,2,3^{*}}$
}

\begin{abstract}
Background: Circulating microRNAs may represent novel markers for cardiovascular diseases. We evaluated whether circulating miRNAs served as potential biomarkers for diffuse myocardial fibrosis in patients with hypertrophic cardiomyopathy ( $\mathrm{HCM})$.

Methods: Cardiac magnetic resonance imaging with postcontrast $T_{1}$ mapping was performed to non-invasively quantify diffuse myocardial fibrosis in HCM patients who were classified into two groups ( $T_{1}<470 \mathrm{~ms}$ or $T_{1} \geq 470 \mathrm{~ms}$, as likely or unlikely to have diffuse fibrosis, respectively). First, we screened 84 miRNAs using human serum/plasma miRNA array on plasma of $8 \mathrm{HCM}$ patients (4/group based on $T_{1}$ time) and 4 healthy controls. From the results of this initial array, 16 miRNAs were selected based on their fold changes and relevance to myocardial fibrosis for further validation by Taqman real-time PCR in 55 HCM patients.

Results: Among the 16 miRNAs, the expression of miR-96-5p and miR-373-3p was low. The remaining 14 (miR18a-5p, miR-146a-5p, miR-30d-5p, miR-17-5p, miR-200a-3p, miR-19b-3p, miR-21-5p, miR-193-5p, miR-10b-5p, miR15a-5p, miR-192-5p, miR-296-5p, miR-29a-3p, and miR-133a-3p) were upregulated in HCM patients with $T_{1}<470$ ms compared with those with $T_{1} \geq 470$ ms, and 11 (except miR-192-5p, miR-296-5p and miR-133a-3p) were significantly inversely correlated with postcontrast $\mathrm{T}_{1}$ values. Individual miRNA had moderate diagnostic value for diffuse myocardial fibrosis (AUC: $0.663-0.742$ ), but the diagnostic value was greatly improved (AUC: 0.87 ) for a combination of 8 miRNAs. In comparison, circulating markers of collagen turnover did not have predictive values for diffuse myocardial fibrosis.
\end{abstract}

Conclusions: These findings suggest that circulating miRNAs provide attractive candidates as putative biomarkers for diffuse myocardial fibrosis in HCM.

Keywords: microRNAs, Myocardial fibrosis, Hypertrophic cardiomyopathy, Cardiac magnetic resonance imaging, Postcontrast $T_{1}$ mapping

\section{Background}

Myocardial fibrosis, a hallmark of various cardiovascular diseases, contributes to heart failure, arrhythmias and sudden death $[1,2]$. Hypertrophic cardiomyopathy

\footnotetext{
*Correspondence: kfanglu@bakeridi.edu.au; a.dart@alfred.org.au 1 Baker IDI Heart and Diabetes Institute, 75 Commercial Road, Melbourne, VIC 3004, Australia

2 Department of Cardiovascular Medicine, Alfred Heart Centre, The Alfred Hospital, 55 Commercial Road, Melbourne, VIC 3004, Australia

Full list of author information is available at the end of the article
}

(HCM) is the most common monogenic cardiac disease and myocardial fibrosis is a common and early feature of HCM [3], associated with the poor prognosis in HCM patients [3, 4].

Historically, myocardial fibrosis could only be definitively diagnosed with cardiac biopsies. There are currently no reliable serological biomarkers to detect myocardial fibrosis. Recent studies have introduced cardiac magnetic resonance imaging (CMR) to noninvasively diagnose myocardial fibrosis [5]. Late gadolinium 
enhancement (LGE) is now an established method to identify regional myocardial fibrosis [6], but it is unable to detect diffuse myocardial fibrosis $[3,6]$. Postcontrast myocardial longitudinal relaxation time $\left(\mathrm{T}_{1}\right)$ mapping is an emerging CMR technique to evaluate diffuse myocardial fibrosis [7]. A number of $\mathrm{T}_{1}$ mapping techniques have been shown to correlate with histologically-quantified fibrosis $[7,8]$. We and others have reported reduced $\mathrm{T}_{1}$ times in several cardiac disease states associated with diffuse fibrosis [7, 9-13]. However, contrast-enhanced CMR is limited by high cost and low availability, and contraindicated in patients with significant renal dysfunction and implanted cardiac devices.

MicroRNAs (miRNAs) are short, noncoding RNAs of 18-25 nucleotides that posttranscriptionally control gene expression by inhibiting protein translation or inducing target mRNA destabilization. miRNAs are powerful regulators of a wide range of important cellular processes and have emerged as dominant players in cardiovascular disease [14]. Several miRNAs, particularly, miR-21, miR29, miR-30, and miR-133, have been implicated in the control of myocardial fibrosis $[15,16]$. miRNAs are also released by cells into circulation [17]. Recent studies have suggested that circulating miRNAs serve as biomarkers for cardiovascular diseases such as acute myocardial infarct, heart failure, coronary artery disease, and hypertension $[18,19]$. However, whether circulating miRNAs can serve as potential biomarkers for myocardial fibrosis has not been evaluated.

In this project, we quantified diffuse myocardial fibrosis using postcontrast $T_{1}$ mapping time. We aimed to study: (1) changes of plasma miRNAs in HCM patients with diffuse myocardial fibrosis indicated by lower $T_{1}$ times, as compared with patients without diffuse fibrosis or healthy controls, (2) the correlations between circulating miRNA levels and postcontrast myocardial $\mathrm{T}_{1}$ times, and diagnostic values of circulating miRNA levels for the detection of diffuse fibrosis.

\section{Methods}

\section{Study population}

We recruited 55 patients referred to the Alfred CMR department for the further evaluation of asymmetric septal hypertrophy (ASH) due to HCM from March 2011 to October 2012. ASH was defined as an interventricular septum thickness of $\geq 15 \mathrm{~mm}$ with a ratio of septal-tolateral ventricular wall thickness of $\geq 1.3: 1.0$ as measured by echocardiography, and the diagnosis of HCM required the absence of any other condition that causes the degree of hypertrophy observed [20]. Exclusion criteria included previous septal reduction therapy, coronary artery disease, atrial fibrillation, valvular heart disease, systemic hypertension, diabetes mellitus, surgery or trauma within previous 6 months, known fibrotic or inflammatory disease or cancer, and contraindications to CMR, including pacemaker and defibrillator implantation, and significant renal dysfunction (estimated glomerular filtration rate (eGFR) $<30 \mathrm{ml} / \mathrm{min} / 1.73 \mathrm{~m}^{2}$ ). This study complied with the Declaration of Helsinki and was approved by the Institutional Ethics Committee of Alfred Healthcare. Informed consent was obtained from all participants. A subset of $8 \mathrm{HCM}$ patients and 4 healthy controls were selected for miRNA array and all $55 \mathrm{HCM}$ patients were included for Taqman real-time PCR analysis.

\section{CMR}

CMR was performed using a clinical 1.5-T scanner (Signa HD 1.5-T, GE Healthcare, Waukesha, Wisconsin, USA). Volumetric LV analysis was performed using the summation of disc method with a contiguous short-axis steady-state free precession pulse sequence stack. LGE was used to identify regional fibrosis using a $\mathrm{T}_{1}$-weighted inversion recovery gradient echo technique, while a $T_{1}$ mapping sequence was used to non-invasively quantify diffuse myocardial fibrosis, as previously described [7, 21]. A region of interest (ROI) was drawn around the entire LV myocardium (excluding papillary muscles) to calculate postcontrast myocardial $\mathrm{T}_{1}$ time. In subjects with regional fibrosis detected by LGE, these areas were excluded from the ROI for the primary analysis of postcontrast myocardial $\mathrm{T}_{1}$ time. $\mathrm{T}_{1}$ times for ROIs including areas of LGE were also calculated. To account for the potential effects of glomerular filtration rate, time delay postcontrast administration, and contrast agent relaxivity on gadolinium pharmacokinetics, corrected values of $\mathrm{T}_{1}$ times were used to normalize postcontrast myocardial $\mathrm{T}_{1}$ times to a matched state (time postcontrast administration $=20 \mathrm{~min}$, eGFR $=90 \mathrm{~mL} / \mathrm{min}$ per $1.73 \mathrm{~m}^{2}$ ) [22]. In addition, raw postcontrast $\mathrm{T}_{1}$ times of the LV blood pool (blood T1 times) were calculated.

\section{Echocardiography}

Transthoracic echocardiography with a standard clinical protocol was performed immediately prior to CMR. Diastolic function was assessed by a combination of mitral inflow pattern ( $E$ to A ratio and deceleration time) and mitral annular velocities $\left(\mathrm{e}^{\prime}\right.$, measured at the septal and lateral aspects of the mitral annulus in the apical 4-chamber view). Additionally, mitral E/e' (septal, lateral and mean) was chosen as an index of LV filling pressure.

\section{Blood sample collection}

Blood samples were obtained before CMR and collected into EDTA-tubes by venepuncture. Plasma samples (10 min centrifugation at $400 \mathrm{~g}$ followed by a further $10 \mathrm{~min}$ at $600 \mathrm{~g}$ ) were stored at $-80{ }^{\circ} \mathrm{C}$ for RNA isolation 
(detailed below). Serum was collected from additional tube without anticoagulants for measurement of amino terminal propeptide of type I and III collagen (PINP and PIIINP) by radioimmunoassay at the Alfred Pathology Department.

\section{Isolation of RNA from plasma samples}

Total RNA was harvested from plasma with Qiazol lysis reagent and miRNeasy mini kit (Qiagen) according to the manufacturers' instructions. $2 \mathrm{ml}$ plasma was aliquoted into 5 eppendorf tubes (400 $\mu \mathrm{l} /$ tube). $400 \mu \mathrm{l}$ plasma was mixed with $1 \mathrm{ml}$ Qiazol lysis reagent, incubated for $5 \mathrm{~min}$, and subsequently mixed with $200 \mu \mathrm{l}$ cholorform for 3 min. After spin, the aqueous phase containing RNA was carefully collected and mixed with $100 \%$ ethanol. RNA was purified by a miRNeasy mini spin column, and eluted by the addition of $30 \mu \mathrm{l}$ RNase-free water. Concentration of RNA was measured by nanodrop, and RNA was stored at $-80{ }^{\circ} \mathrm{C}$ for further processing.

\section{miScript II RT kit and miScript miRNA PCR array}

$0.5 \mu \mathrm{g}$ total RNA was reversely transcribed into cDNA using the miScript II RT kit (Qiagen, Netherlands). Then, cDNA was mixed with miScript SyBR green qPCR mastermix (Qiagen). $25 \mu \mathrm{l}$ of the cocktail was aliquoted into each well of 96 well plates containing the pre-dispensed miRNA-specific assays [Human Serum/plasma miRNA PCR Array, SABiosciences (Qiagen)]. PCR and data analysis was performed on an ABI Prism 7300 system (Applied Biosystems, USA). The transcript abundance was expressed as fold change over the value of the healthy control group calculated by $2^{-\Delta \Delta \mathrm{Ct}}$ method. Eight housekeeping genes including miR-39 and U6 were used. Since U6 was quite consistent among the three groups among eight housekeeping genes, U6 was chosen as the reference miRNA for validation study by real-time PCR.

\section{Taqman real-time PCR}

To confirm data from miScript miRNA PCR array, we measured the expression of several dysregualated miRNAs using Taqman real-time PCR (Applied Biosystems). $5 \mu \mathrm{l}$ RNA (10 ng RNA) was reversely transcribed into cDNA using Taqman miRNA reverse transcription kit (Applied Biosystems). PCR products were then amplified from cDNA samples using the Taqman miRNA assay together with Taqman Universal PCR master mix (Applied Biosystems). The reaction volume was $20 \mu \mathrm{l}$. PCR reactions were performed on an ABI Prism 7500 system (Applied Biosystems). miRNAs of each group were expressed as relative miRNA expression levels using $\mathrm{U} 6$ as a reference value by calculating $2^{-\Delta \mathrm{Ct}}$ i.e. $2^{-\mathrm{Ct}(\mathrm{miRNA})-\mathrm{Ct}(\mathrm{U} 6)}$.

\section{Statistical analysis}

Data were expressed as mean \pm SD unless otherwise stated. SPSS 17.0 was used for statistical analysis. The normality of data was tested by Kolmogorov-Smirnov test. Chi square test was used to compare discrete variables among groups. For miRNA array data, to compare differences among 3 groups, one-way ANOVA followed by Tukey multiple comparison test and Kruskal-Wallis test were used for parametic and nonparametic data, respectively. Student $t$ test or Mann-Whitney $U$ test was employed for comparison between the two subgroups of $55 \mathrm{HCM}$ patients when appropriate. Spearman correlation coefficients were computed to assess the correlations between postcontrast $\mathrm{T}_{1}$ times and miRNAs. Receiver operating characteristic (ROC) curve analysis was used to calculate the area under the curve (AUC) of individual miRNA for diagnosing diffuse fibrosis. To calculate the predictive value of multiple miRNAs for diffuse fibrosis, a logistic regression model using backward stepwise (likelihood ratio) method was used to calculate predicted probabilities. Skewed data was Ln transformed prior to inclusion in the logistic regression model. AUC was then calculated using ROC curve analysis performed on predicted probabilities. A difference of $P<0.05$ (two-sided) was considered statistically significant.

\section{Results}

\section{Patient demographics}

Patient demographics, CMR and echocardiograph data for miRNA array $(n=12)$ and real-time PCR $(n=55)$ are presented in Tables 1 and 2, respectively. The normal range of postcontrast myocardial $\mathrm{T}_{1}$ times in healthy controls in our previous study was $561 \pm 47 \mathrm{~ms}$ [11], so $\mathrm{T}_{1}$ time (excluding regions of LGE) of $470 \mathrm{~ms}$ (approximately $2 \mathrm{SD}$ below the mean of control values) was chosen to divide the HCM cohort into those likely $(<470 \mathrm{~ms})$ and unlikely $(\geq 470 \mathrm{~ms})$ to have significant diffuse myocardial fibrosis [21]. Postcontrast myocardial $\mathrm{T}_{1}$ times excluding LGE and including LGE were shown in Tables 1 and 2. $T_{1}$ times corrected for GFR and time delay postcontrast administration were also calculated and shown. There was no significant difference in raw blood $T_{1}$ times between groups, effectively ruling out contrast kinetics as a confounding factor for the observed differences in myocardial $\mathrm{T}_{1}$ time between groups. LGE (regional fibrosis) was observed in the majority of HCM patients and the mean quantity of LGE did not differ significantly between the $2 \mathrm{HCM}$ groups (Tables 1,2). For miRNA array study, lateral and mean $\mathrm{e}^{\prime}$ were significantly lower in $\mathrm{HCM}$ patients compared to controls. Lateral and mean $\mathrm{E} / \mathrm{e}^{\prime}$ were significantly higher in patients with diffuse fibrosis compared to controls. As expected, septal thickness and the ratio of septal to 
Table 1 Subject characteristics for miRNA array

\begin{tabular}{|c|c|c|c|}
\hline & Control & $\mathrm{T}_{1} \geq 470$ & $\mathrm{~T}_{1}<470$ \\
\hline$n$ & 4 & 4 & 4 \\
\hline Gender (m/f) & $4 / 0$ & $3 / 1$ & $2 / 2$ \\
\hline Age (years) & $42 \pm 16.3$ & $49.5 \pm 5.5$ & $58.3 \pm 9.5$ \\
\hline Height (cm) & $175.8 \pm 8.6$ & $172.8 \pm 5.9$ & $165.5 \pm 11.1$ \\
\hline Weight (kg) & $77.8 \pm 4.1$ & $83.5 \pm 20.3$ & $70 \pm 6.7$ \\
\hline Body mass index $\left(\mathrm{kg} / \mathrm{m}^{2}\right)$ & $25.4 \pm 3.5$ & $27.8 \pm 5.8$ & $25.8 \pm 4$ \\
\hline Family history of HCM (\%) & NA & $50 \%$ & $75 \%$ \\
\hline Resting heart beat (beats/min) & $66.8 \pm 9.5$ & $69 \pm 11.6$ & $60.5 \pm 7.9$ \\
\hline Systolic blood pressure (mmHg) & $120.4 \pm 10.2$ & $129.5 \pm 16.6$ & $125.3 \pm 11.6$ \\
\hline Diastolic blood pressure $(\mathrm{mmHg})$ & $73 \pm 9.8$ & $77.3 \pm 10.8$ & $70 \pm 10.9$ \\
\hline $\mathrm{eGFR}\left(\mathrm{mL} / \mathrm{min} / 1.75 \mathrm{~m}^{2}\right)$ & $87.8 \pm 6.5$ & $79 \pm 10.7$ & $77.3 \pm 15$ \\
\hline \multicolumn{4}{|l|}{ Medications } \\
\hline$\beta$-blockers & NA & $25 \%$ & $75 \%$ \\
\hline Calcium channel blockers & NA & $25 \%$ & $25 \%$ \\
\hline Angiotensin convert enzyme inhibitor & NA & $0 \%$ & $0 \%$ \\
\hline Angiotensin receptor blockers & NA & $50 \%$ & $0 \%^{\dagger}$ \\
\hline \multicolumn{4}{|l|}{ Echocardiography } \\
\hline Left atrial volume indexed, $\mathrm{ml} / \mathrm{m}^{2}$ & $34.2 \pm 10.2$ & $43.1 \pm 10.4$ & $56.9 \pm 21.3$ \\
\hline E/A ratio & $1.53 \pm 0.48$ & $1.16 \pm 0.44$ & $1.38 \pm 0.51$ \\
\hline Deceleration time (ms) & $158.5 \pm 15.6$ & $226.8 \pm 44.8^{*}$ & $200.3 \pm 25.2$ \\
\hline Septal e' $(\mathrm{cm} / \mathrm{s})$ & $10 \pm 3.6$ & $6.2 \pm 2.1$ & $6 \pm 2.2$ \\
\hline Lateral e' (cm/s) & $13.3 \pm 2.3$ & $7.9 \pm 3^{*}$ & $7.8 \pm 2.9^{*}$ \\
\hline Mean $e^{\prime}(\mathrm{cm} / \mathrm{s})$ & $11.7 \pm 2.9$ & $7 \pm 2.5^{*}$ & $6.9 \pm 2.3^{*}$ \\
\hline Septal E/e' & $8.6 \pm 3.4$ & $11.1 \pm 2.8$ & $18.9 \pm 8.8$ \\
\hline Lateral E/e' & $6 \pm 1.3$ & $9 \pm 2.9$ & $14.1 \pm 5.9^{*}$ \\
\hline Mean E/e' & $7.3 \pm 2.3$ & $10.1 \pm 2.9$ & $16.5 \pm 7.2^{*}$ \\
\hline Resting LVOT gradient $(\mathrm{mmHg})$ & $4.5 \pm 1.1$ & $47.8 \pm 68.7$ & $51.6 \pm 60$ \\
\hline \multicolumn{4}{|l|}{ CMR } \\
\hline $\mathrm{T}_{1}$ times (ms), excluding LGE & $578.5 \pm 42$ & $561.5 \pm 31.9$ & $440.3 \pm 30.3^{* *,+\dagger}$ \\
\hline$T_{1}$ times (ms), including LGE & $578.5 \pm 42$ & $550.9 \pm 44.6$ & $435.3 \pm 45.5^{* * *,+\dagger}$ \\
\hline $\mathrm{T}_{1}$ times (ms, corrected values) & $567.6 \pm 50.6$ & $549.7 \pm 34.7$ & $422.4 \pm 26.6^{* *,+\dagger}$ \\
\hline Blood $T_{1}$ times $(\mathrm{ms})$ & $296.0 \pm 5.3$ & $300.8 \pm 31.7$ & $302.3 \pm 18.8$ \\
\hline Septal thickness (mm) & $7.75 \pm 0.96$ & $18 \pm 3.6^{* *}$ & $19 \pm 3.2^{* *}$ \\
\hline Lateral wall thickness (mm) & $7.5 \pm 1$ & $8.5 \pm 1.3$ & $8.3 \pm 1.7$ \\
\hline Septal/lateral wall thickness & $1.04 \pm 0.07$ & $2.12 \pm 0.35^{* *}$ & $2.35 \pm 0.44^{* *}$ \\
\hline LV mass index (g/BSA) & $55.1 \pm 8.9$ & $90.6 \pm 30.4$ & $94.0 \pm 28.3$ \\
\hline LV ejection fraction (\%) & $57.5 \pm 6.1$ & $69 \pm 2.9$ & $67 \pm 15.5$ \\
\hline Presence of LGE (\%) & NA & $75 \%$ & $75 \%$ \\
\hline Quantity of LGE (\% of LV) mass & NA & $2.9 \pm 4$ & $10.1 \pm 12.2$ \\
\hline
\end{tabular}

Data are expressed as mean \pm SD

$C M R$ cardiac magnetic resonance, $L V$ left ventricular, $L V O T$ left ventricular outflow tract, $L G E$ late gadolinium enhancement, $B S A$ body surface area, $T_{1}$ times (corrected values): $T_{1}$ times were normalized to a matched state (time post-contrast administration $=20$ min, eGFR=90 mL/min $/ 1.73 \mathrm{~m}{ }^{2}$ ) to account for the potential effects of glomerular filtration rate, time delay post-contrast administration, and contrast agent relaxivity on gadolinium pharmacokinetics

${ }^{*} P<0.05,{ }^{* *} P<0.01,{ }^{* * *} P<0.001$, vs. controls, ${ }^{\dagger} P<0.05,{ }^{+\dagger} P<0.001$, vs. $\mathrm{T}_{1} \geq 470 \mathrm{~ms}$

lateral wall thickness, were significantly increased in both HCM groups compared with controls (Table 1). For real-time PCR validation study, the presence of family history was significantly higher in patients without diffuse fibrosis compared to those with diffuse fibrosis
(Table 2). For both miRNA array and real-time PCR study (Tables 1, 2), there were no significant differences in age, gender, BMI, eGFR, heart rate, and blood pressure among groups. HCM groups had normal systolic function (LVEF). 
Table 2 Subject characteristics for real-time PCR

\begin{tabular}{|c|c|c|}
\hline & $\mathrm{T}_{1} \geq 470$ & $\mathrm{~T}_{1}<470$ \\
\hline$n$ & 28 & 27 \\
\hline Gender (m/f) & $22 / 6$ & $22 / 5$ \\
\hline Age (years) & $49.1 \pm 14.0$ & $49.9 \pm 10.8$ \\
\hline Height (cm) & $171.3 \pm 10.7$ & $174.9 \pm 7.9$ \\
\hline Weight (kg) & $81.8 \pm 18.7$ & $88.5 \pm 14.7$ \\
\hline Body mass index $\left(\mathrm{kg} / \mathrm{m}^{2}\right)$ & $27.6 \pm 4.5$ & $28.9 \pm 4.0$ \\
\hline Family history of HCM (\%) & $35.7 \%$ & $11.1 \% *$ \\
\hline Resting heart beat (beats/min) & $63.9 \pm 10.5$ & $61.5 \pm 16.1$ \\
\hline Systolic blood pressure (mmHg) & $129.7 \pm 17.3$ & $130.8 \pm 14.9$ \\
\hline Diastolic blood pressure (mmHg) & $75.0 \pm 9.1$ & $71.6 \pm 8.6$ \\
\hline $\mathrm{eGFR}\left(\mathrm{mL} / \mathrm{min} / 1.75 \mathrm{~m}^{2}\right)$ & $83.9 \pm 7.6$ & $79.7 \pm 13.8$ \\
\hline \multicolumn{3}{|l|}{ Medications } \\
\hline$\beta$-blockers & $50 \%$ & $63 \%$ \\
\hline Calcium channel blockers & $14.3 \%$ & $29.6 \%$ \\
\hline Angiotensin convert enzyme inhibitor & $10.7 \%$ & $22.2 \%$ \\
\hline Angiotensin receptor blockers & $21.4 \%$ & $3.7 \%$ \\
\hline \multicolumn{3}{|l|}{ Echocardiography } \\
\hline Left atrial volume indexed, $\mathrm{ml} / \mathrm{m}^{2}$ & $48.4 \pm 18.2$ & $49.6 \pm 13.9$ \\
\hline E/A ratio & $1.35 \pm 0.63$ & $1.40 \pm 0.67$ \\
\hline Deceleration time (ms) & $200.0 \pm 64.0$ & $226.4 \pm 44.0$ \\
\hline Septal e' (cm/s) & $6.5 \pm 1.5$ & $5.9 \pm 1.7$ \\
\hline Lateral e' (cm/s) & $8.5 \pm 1.9$ & $7.6 \pm 2.9$ \\
\hline Mean $\mathrm{e}^{\prime}(\mathrm{cm} / \mathrm{s})$ & $7.5 \pm 1.4$ & $6.7 \pm 2.1$ \\
\hline Septal E/e' & $12.5 \pm 3.8$ & $14.8 \pm 5.9$ \\
\hline Lateral E/e' & $9.8 \pm 3.8$ & $12.0 \pm 5.1$ \\
\hline Mean E/e' & $11.0 \pm 3.4$ & $13.4 \pm 5.1$ \\
\hline Resting LVOT gradient (mmHg) & $32.8 \pm 39.6$ & $49.9 \pm 51.0$ \\
\hline \multicolumn{3}{|l|}{ CMR } \\
\hline $\mathrm{T}_{1}$ times (ms), excluding LGE & $536.3 \pm 54.6$ & $441.0 \pm 30.0^{* * *}$ \\
\hline $\mathrm{T}_{1}$ times (ms), including LGE & $521.3 \pm 66.4$ & $432.3 \pm 26.2^{* * *}$ \\
\hline $\mathrm{T}_{1}$ times (ms, corrected values) & $518.7 \pm 55.2$ & $423.8 \pm 34.1^{* * *}$ \\
\hline Blood $T_{1}$ times $(\mathrm{ms})$ & $310.7 \pm 30.4$ & $296.0 \pm 29.3$ \\
\hline Septal thickness (mm) & $20.3 \pm 5.8$ & $19.1 \pm 3.3$ \\
\hline Lateral wall thickness (mm) & $8.6 \pm 1.8$ & $9.2 \pm 1.9$ \\
\hline Septal/lateral wall thickness & $2.4 \pm 0.7$ & $2.1 \pm 0.5$ \\
\hline LV mass index (g/BSA) & $92.6 \pm 35.0$ & $87.4 \pm 22.4$ \\
\hline LV ejection fraction (\%) & $68.5 \pm 7.3$ & $70.8 \pm 6.5$ \\
\hline Presence of LGE (\%) & $85.7 \%$ & $81.5 \%$ \\
\hline Quantity of LGE (\% of LV) mass & $5.6 \pm 7.5$ & $4.7 \pm 6.1$ \\
\hline
\end{tabular}

Data are expressed as mean \pm SD

$C M R$ cardiac magnetic resonance, $L V$ left ventricular, $L V O T$ left ventricular outflow tract, $L G E$ late gadolinium enhancement, $B S A$ body surface area, $T_{1}$ times (corrected values): $\mathrm{T}_{1}$ times were normalized to a matched state (time post-contrast administration $=20 \mathrm{~min}$, eGFR $=90 \mathrm{~mL} / \mathrm{min} / 1.73 \mathrm{~m}{ }^{2}$ ) to account for the potential effects of glomerular filtration rate, time delay post-contrast administration, and contrast agent relaxivity on gadolinium pharmacokinetics

${ }^{*} P<0.05$, ${ }^{* *} P<0.001$, vs. $\mathrm{T}_{1} \geq 470 \mathrm{~ms}$

miRNA PCR array

Human serum/plasma miRNA PCR array was performed to measure 84 miRNAs in plasma samples from $8 \mathrm{HCM}$ patients and 4 controls (Additional file 1). Plasma levels of 14 miRNAs significantly differed among the 3 groups, and these 14 miRNAs also significantly increased in patients with diffuse fibrosis compared to healthy controls (all $>5$ folds) (Fig. 1). 


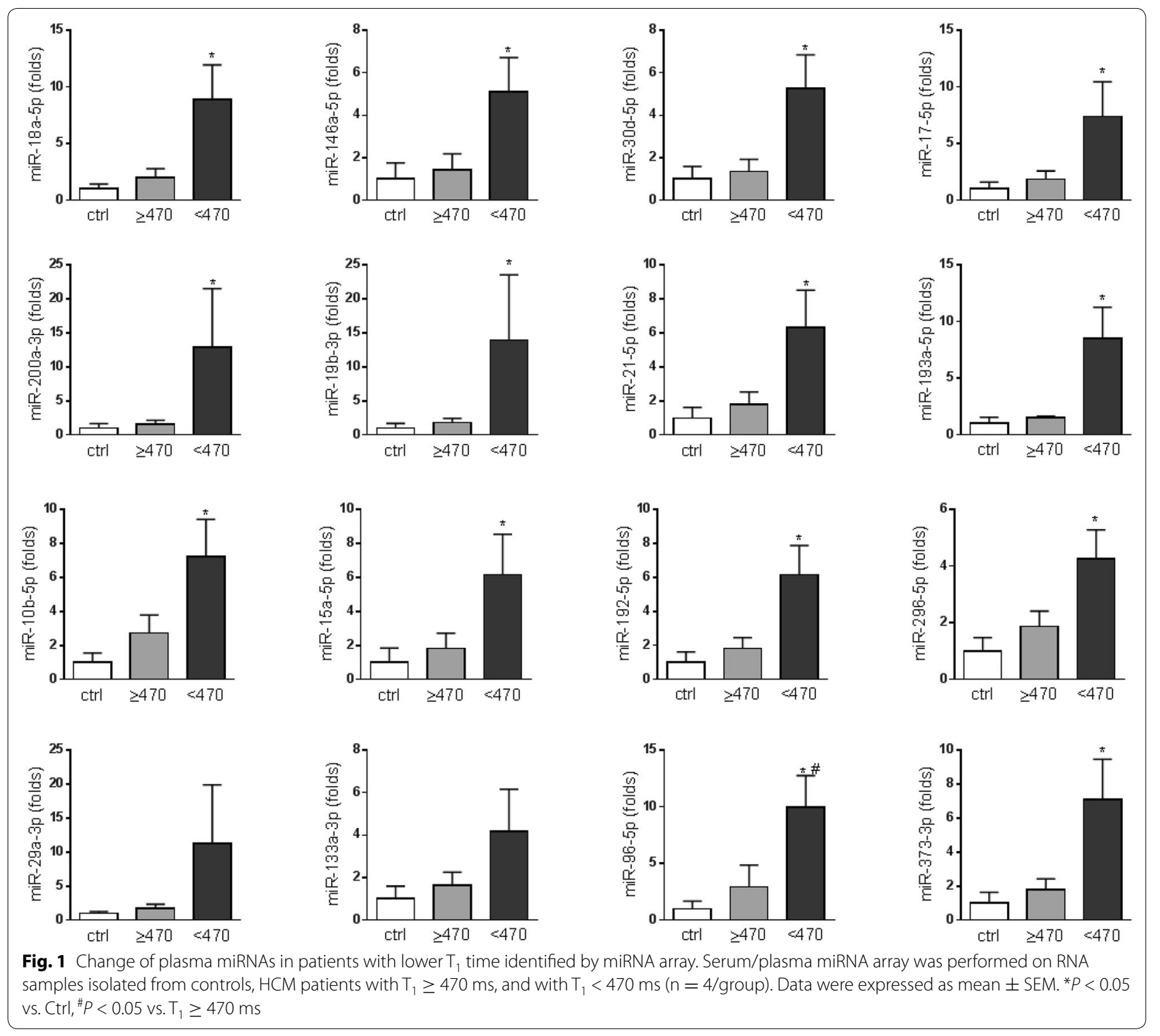

The upregulation in patients with diffuse fibrosis did not reach significance compared to patients without diffuse fibrosis except for miR-96-5p due to small sample size $(\mathrm{n}=4 /$ group$)$. Furthermore, levels of these 14 miRNAs were negatively correlated with postcontrast $\mathrm{T}_{1}$ times ( 9 of them have $\mathrm{r}<-0.5,5$ of them have $\mathrm{r}<-0.4$, Table 3$)$. These 14 miRNAs were further validated in $55 \mathrm{HCM}$ patients. Interestingly, miR-29a-3p and miR-133a-3p, known to be involved in myocardial fibrosis, were not significantly different among 3 groups (Fig. 1). We included miR-29a-3p and miR-133a-3p for further validation since their roles in myocardial fibrosis are well established.
Validation of miRNA PCR array by real-time PCR

We validated the expression of the above 14 miRNAs plus miR-29a-3p and miR-133a-3p in all 55 HCM patients by Taqman real-time PCR. Of the 14 miRNAs that significantly differed among the 3 groups by miRNA array, 12 miRNAs were confirmed to be significantly upregulated in patients with diffuse fibrosis compared with those without diffuse fibrosis (Fig. 2). Notably, the expression of miR-96-5p and miR-373-3p was very low (data not shown). miR-29a-3p and miR-133a-3p were also significantly increased in patients with diffuse fibrosis (Fig. 2). $11 \mathrm{miRNAs}$ were significantly and inversely correlated with postcontrast $\mathrm{T}_{1}$ times, but the inverse correlations 
Table 3 Correlations between circulating miRNAs measured by miRNA array and $T_{1}$ times

\begin{tabular}{lll}
\hline miRNA & $\mathbf{r}$ & $\boldsymbol{P}$ value \\
\hline miR-18a-5p & -0.521 & 0.082 \\
miR-146a-5p & -0.658 & 0.020 \\
miR-30d-5p & -0.599 & 0.040 \\
miR-17-5p & -0.458 & 0.134 \\
miR-200a-3p & -0.436 & 0.157 \\
miR-19b-3p & -0.434 & 0.159 \\
miR-21-5p & -0.443 & 0.150 \\
miR-193a-5p & -0.553 & 0.062 \\
miR-10b-5p & -0.548 & 0.065 \\
miR-15a-5p & -0.475 & 0.119 \\
miR-192-5p & -0.512 & 0.089 \\
miR-296-5p & -0.557 & 0.060 \\
miR-96-5p & -0.579 & 0.049 \\
miR-373-3p & -0.517 & 0.085 \\
\hline
\end{tabular}

Spearman correlation coefficients were computed to assess the correlations between postcontrast $\mathrm{T} 1$ times and miRNAs

with $\mathrm{T}_{1}$ times were not significant for miR-192-5p $(\mathrm{r}=0.246, \mathrm{p}=0.071), \mathrm{miR}-296-5 \mathrm{p}(\mathrm{r}=0.239, \mathrm{p}=0.079)$ and miR-133a-3p $(\mathrm{r}=-0.208, P=0.127)$ (Fig. 3). Circulating miRNA levels were not correlated with the prevalence or the mean quantity of regional fibrosis quantified by LGE, or basic demographic characteristics (age, gender, BMI, blood pressure, family history of $\mathrm{HCM}$, and left ventricular ejection fraction).

\section{Diagnostic values of plasma miRNAs for detection of diffuse myocardial fibrosis}

The predictive power of circulating miRNAs to identify diffuse myocardial fibrosis was evaluated by ROC curve analysis. Individual ROCs of 14 miRNAs including miR-29a-3p and miR-133-3p showed moderate predictive values for the presence of diffuse myocardial fibrosis (AUC ranges from 0.663 to 0.742 , Table 4 ). We then examined whether a complex of miRNAs had improved predictive values over single miRNA for detection of diffuse fibrosis by using logistic regression analysis. AUC for combination of all 14 miRNAs reached 0.87 . To avoid overfitting of the data due to redundancies among 14 miRNAs, a logistic regression model with backward stepwise (likelihood ratio) method was employed. 8 miRNAs (miR-18a-5p, miR-30d-5p, miR-21-5p, miR193-5p, miR-10b-5p, miR-15a-5p, miR-296-5p, and miR29a-3p) were selected by the model and the AUC for the combination of these 8 miRNAs remained 0.87 (Fig. 4).

\section{Circulating markers of collagen turnover}

PINP and PIIINP are markers reflecting the status of collagen turnover. Neither PINP nor PIIINP significantly differed between patients with or without diffuse fibrosis (Fig. 2). AUC of PINP and PIIINP was 0.588 and 0.540, respectively (both $P>0.05$ ).

\section{Discussion}

Circulating miRNAs are potential biomarkers for various cardiovascular diseases $[18,19]$. In the present study, we aimed to evaluate whether circulating miRNAs can serve as potential biomarkers for myocardial fibrosis in $\mathrm{HCM}$ patients. CMR postcontrast $\mathrm{T}_{1}$ mapping was used to separate HCM patients into 2 subgroups $\left(\mathrm{T}_{1}<470 \mathrm{~ms}\right.$ or $\mathrm{T}_{1} \geq 470 \mathrm{~ms}$ as likely or unlikely to have diffuse myocardial fibrosis, respectively). We identified 14 miRNAs which significantly differed among the 3 groups $\left(\mathrm{T}_{1}<470 \mathrm{~ms}, \mathrm{~T}_{1} \geq 470 \mathrm{~ms}\right.$ and controls, $4 /$ group) using human serum/plasma miRNA array. We then validated the above 14 miRNAs plus miR-29a-3p and miR-133a-3p (known to be involved in fibrosis) using Taqman real-time PCR in 55 HCM patients. Out of 16 miRNAs selected, 14 were confirmed to be elevated in HCM patients with $\mathrm{T}_{1}<470 \mathrm{~ms}$ compared to those with $\mathrm{T}_{1} \geq 470 \mathrm{~ms}$, while $11 \mathrm{miRNA}$ levels significantly and inversely correlated with postcontrast $\mathrm{T}_{1}$ times. Furthermore, individual ROCs of these 14 miRNAs showed moderate predictive values for the presence of diffuse myocardial fibrosis (AUC ranges from 0.663 to 0.742 ), but a combination of miRNAs has good diagnostic value for diffuse myocardial fibrosis with an AUC of 0.87. Our results suggest that circulating miRNAs represent a novel circulating marker of diffuse myocardial fibrosis.

Myocardial fibrosis is difficult to diagnose noninvasively. Recent studies have introduced CMR to noninvasively diagnose myocardial fibrosis. However, the use of CMR is limited by its high cost and contraindications, and low availability of CMR facility and expertise. Circulating miRNAs could be practical and attractive markers for myocardial fibrosis since they are easily accessible, reliably stable and disease-specific. In the present study, we have demonstrated that circulating miRNAs are associated with diffuse fibrosis in HCM patients. Previous studies have suggested that peripheral collagen markers such as PINP and PIIIP may serve as markers for myocardial fibrosis. Circulating collagen I synthesis marker was strongly correlated with myocardial fibrosis in hypertensive patients [23], and higher circulating PIIINP concentrations were associated with increased cardiovascular mortality [24]. However, other studies did not show correlations between collagen markers (PINP, PIIINP) and myocardial fibrosis in patients with aortic stenosis [25]. Therefore, we were interested to know whether PINP and PIIINP are markers for myocardial fibrosis in patients with HCM. PINP and PIIINP did not increase in diffuse fibrosis with an AUC $=0.588$ and 0.540 , respectively. 


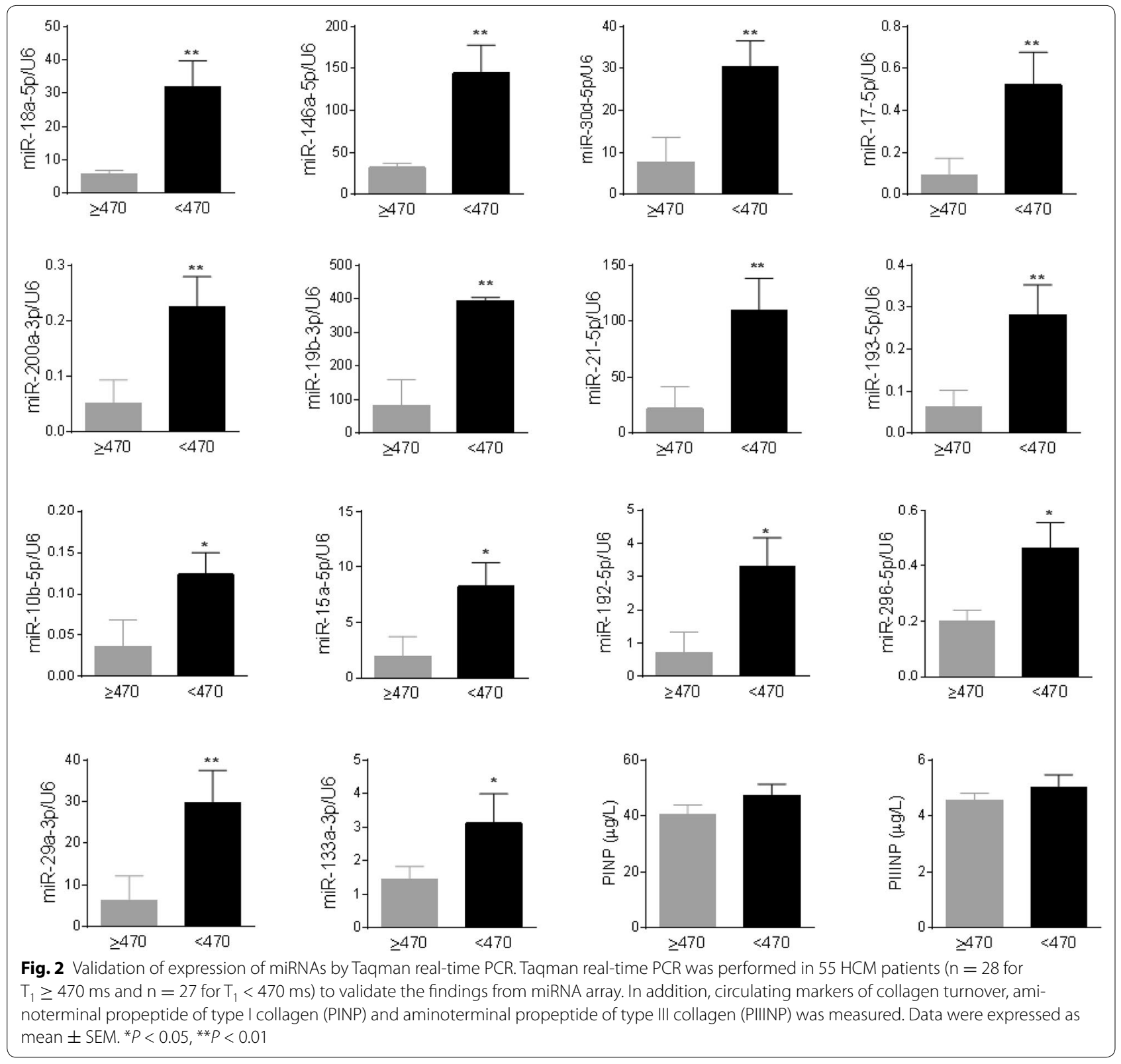

So, existing collagen turnover markers cannot be used in the diagnosis of diffuse fibrosis in HCM patients. In a recently published paper, the authors evaluated the correlation between circulating miRNAs and regional fibrosis quantified by LGE in HCM patients, and they found that miR-29a significantly correlated with regional fibrosis [26]. However, they did not quantified diffuse fibrosis in HCM patients. In the current study, we did not find significant correlations between miRNAs and regional fibrosis. Our study focused on the correlation of miRNAs with diffuse fibrosis and we found that $14 \mathrm{miR}$ NAs including miR-29a-3p were upregulated in diffuse fibrosis. Our group has recently demonstrated that the amount of diffuse fibrosis quantified by postcontrast $T_{1}$ mapping correlate with invasively demonstrated left ventricular stiffness in cardiac transplant recipients [27]. So, in HCM patients with CMR evidence of diffuse fibrosis, increased ventricular stiffness could contribute to diastolic heart failure.

Among 14 miRNAs identified in our study, the roles of miR-21, miR-29a, miR-30d and miR-133a in myocardial fibrosis are well established. miR-21, one of the most widely investigated miRNA, regulates fibroblast survival and promotes fibrosis through targeting sprouty/ 


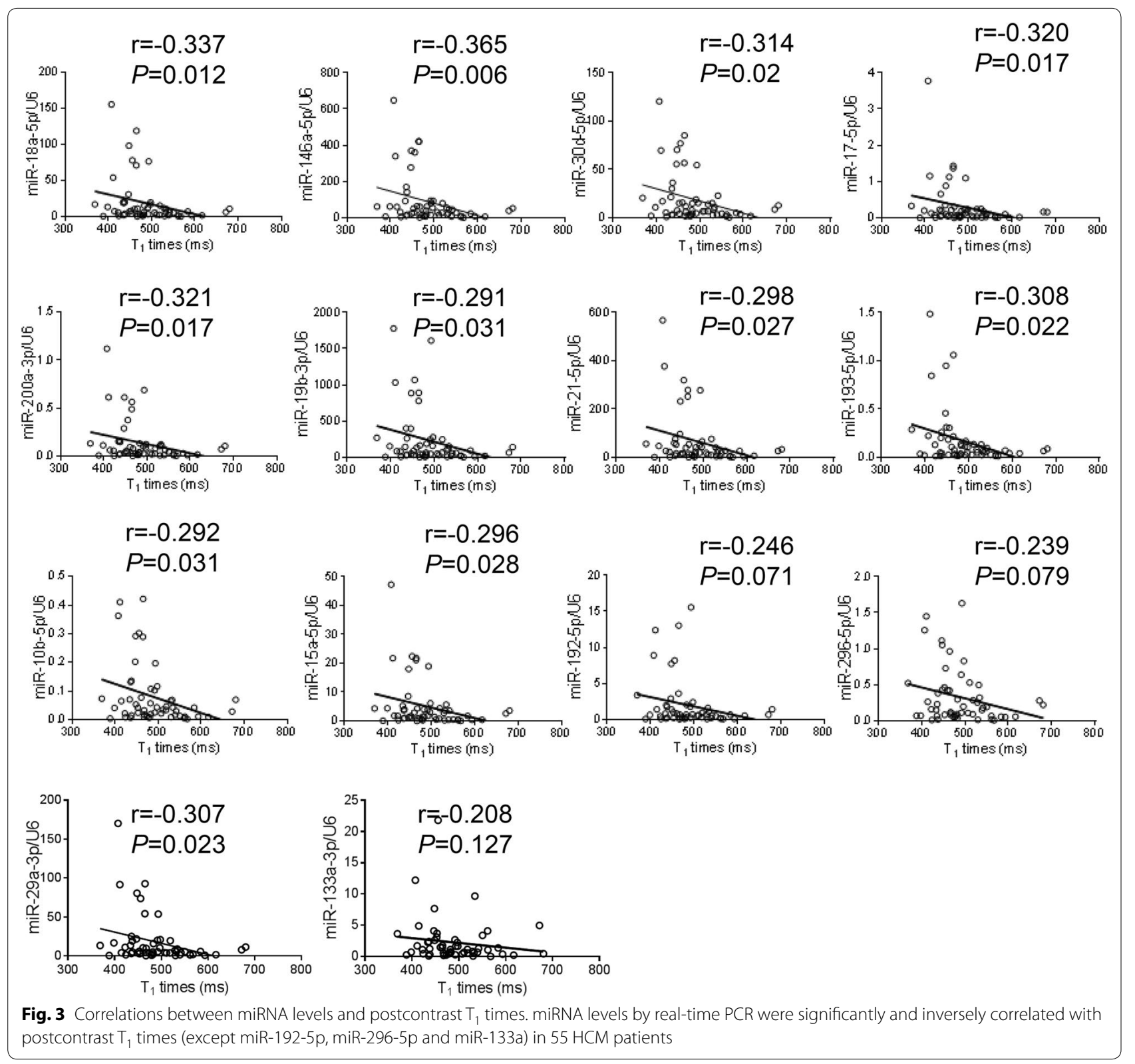

ERK pathways [28]. miR-29 is the best characterized direct regulator of extracelluar matrix protein synthesis [29], while miR-30 and miR-133a target connective tissue growth factor (CTGF) [30]. miR-17-92 cluster (miR17, miR-18a, miR-19a, and miR-19b), also target CTGF as well as thrombospondin-1 in the context of myocardial fibrosis [31]. miR-192 and miR-200a are involved in transforming growth factor (TGF) $\beta$ signaling [32, 33]. Epithelial-to-mesenchymal transition (EMT) is another mechanism mediated by miRNAs in myocardial fibrosis and miR-10b, miR-192 and miR-200a have a role in TGF$\beta$-dependent EMT [32-35]. miR-146a is an important regulator of the immune response and inflammation $[36,37]$. In addition, miR-15a is essential for apoptosis [38], while miR-296 has been named an angiomiR [39]. However, the role of miR-193 in fibrosis is unclear. Taken together, the miRNAs identified in our study are involved in myocardial fibrosis through different mechanisms such as regulating TGF- $\beta$ /CTGF signaling pathway, ECM proteins, fibroblasts and EMT.

It seems that the elevation of circulating miRNAs in HCM patients with myocardial fibrosis is caused by its upregulation in the stressed myocardium. HCM is characterized by mutations in sarcomeric proteins 
Table 4 AUC for individual miRNA

\begin{tabular}{lllcll}
\hline & AUC & P value & Cutoff & $\begin{array}{l}\text { Sensitivity } \\
(\%)\end{array}$ & $\begin{array}{l}\text { Specificity } \\
(\%)\end{array}$ \\
\hline miR-18a-5p & 0.742 & 0.002 & 6.65 & 70.4 & 71.4 \\
miR-146a-5p & 0.737 & 0.003 & 50.04 & 69.6 & 78.6 \\
miR-30d-5p & 0.729 & 0.004 & 15.19 & 65.2 & 84.4 \\
miR-17-5p & 0.722 & 0.005 & 0.16 & 60.9 & 71.9 \\
miR-200a-3p & 0.721 & 0.005 & 0.085 & 60.9 & 75 \\
miR-19b-3p & 0.712 & 0.007 & 127.38 & 59.3 & 78.6 \\
miR-21-5p & 0.710 & 0.007 & 37.18 & 59.3 & 82.1 \\
miR-193-5p & 0.709 & 0.008 & 0.11 & 59.3 & 82.1 \\
miR-10b-5p & 0.701 & 0.010 & 0.05 & 60.9 & 75 \\
miR-15a-5p & 0.694 & 0.013 & 2.88 & 59.3 & 71.4 \\
miR-192-5p & 0.681 & 0.021 & 0.72 & 63 & 75 \\
miR-296-5p & 0.681 & 0.021 & 0.26 & 56.5 & 71.4 \\
miR-29a-3p & 0.717 & 0.006 & 9.31 & 63 & 82.1 \\
miR-133a-3p & 0.663 & 0.038 & 1.42 & 63 & 78.6 \\
\hline Receiver operating & & & & 75 \\
\hline
\end{tabular}

Receiver operating characteristic (ROC) curve analysis was used to calculate the area under the curve (AUC) of individual miRNA for diagnosing diffuse fibrosis

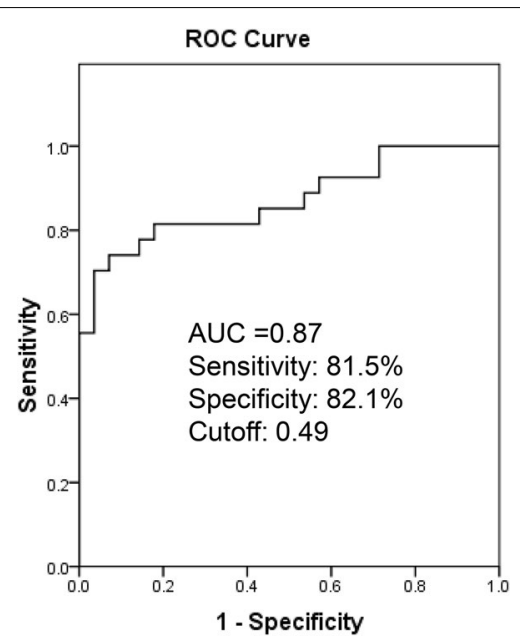

Fig. 4 ROC analysis of the complex of 8 miRNAs (miR-18a-5p, miR30d-5p, miR-21-5p, miR-193-5p, miR-10b-5p, miR-15a-5p, miR-296-5p, and miR-29a-3p) was used to predict diffuse fibrosis in HCM patients

of cardiomyocytes. Cardiac fibroblasts are activated through the interactions between cardiomyocytes and cardiac fibroblasts. miR-21 and miR-29a are fibroblastenriched, while miR-133a is cardiomyocyte-enriched. miR-30d comes from both cardiomyocytes and cardiac fibroblasts. Circulating miRNAs may also come from other cell types such as endothelial cells and immune cells. For example, miR-17-92 cluster are expressed on endothelial cells in addition to cardiomyocytes [40], and miR-296, miR-10b, miR-192 and miR-15a are also expressed on endothelial cells [41]. miR-146a is abundant in immune cells in addition to its expression in the heart [42] and miR-193 is also expressed on mononuclear cells [43]. It is speculated that cardiomyocytes and fibroblasts under stress may send signals to other cell types, and induce the release of miRNAs from other cells into circulation. In addition, miRNAs are potential players in such intercellular communication $[19,44]$ by directly acting as paracrine signals or by modulating downstream intercellular signaling mediators.

\section{Conclusion}

We have demonstrated that 14 circulating miRNAs are associated with diffuse myocardial fibrosis quantified by postcontrast $\mathrm{T}_{1}$ mapping. Although individual miRNA has moderate diagnostic value for diffuse fibrosis, the diagnostic power is greatly improved (AUC: 0.87 ) for a combination of miRNAs. Thus, circulating miRNAs could be a favourable alternative to CMR for assessing diffuse myocardial fibrosis because of its convenience, with no contraindications.

\section{Additional file}

Additional file 1. Human plasma/serum miRNA PCR array data.

\section{Abbreviations}

HCM: hypertrophic cardiomyopathy; miR: microRNA; CMR: cardiac magnetic resonance imaging; LGE: late gadolinium enhancement; $A S H$ : asymmetric septal hypertrophy; eGFR: estimated glomerular filtration rate; ROI: region of interest; LV: left ventricular; ROC: receiver operating characteristic; AUC: area under the curve; EMT: epithelial-to-mesenchymal transition; TGF- $\beta$ : transforming growth factor- $\beta$; CTGF: connective tissue growth factor.

\section{Authors' contributions}

LF designed the study, performed experiments, analysed data and wrote the paper. AE recruited patients, performed CMR and contributed to the paper. XM performed experiments and contributed to the paper. DW performed experiments and contributed to the paper. AT performed CMR and contributed to the paper. JCD contributed to the design of the study and the paper. AD designed the study, analysed data and contributed to the paper. All authors read and approved the final manuscript.

\section{Author details}

${ }^{1}$ Baker IDI Heart and Diabetes Institute, 75 Commercial Road, Melbourne, VIC 3004, Australia. ${ }^{2}$ Department of Cardiovascular Medicine, Alfred Heart Centre, The Alfred Hospital, 55 Commercial Road, Melbourne, VIC 3004, Australia.

${ }^{3}$ Monash University, Melbourne, Australia.

\section{Acknowledgements}

This study was supported in part by the Victorian Government's Operational Infrastructure Support Program. Dr Dart is a NHMRC fellow.

\section{Compliance with ethical guidelines}

\section{Competing interests}

The authors declare that they have no competing interests.

Received: 25 March 2015 Accepted: 14 September 2015

Published online: 24 September 2015 


\section{References}

1. Zile MR, Brutsaert DL. New concepts in diastolic dysfunction and diastolic heart failure: Part II: causal mechanisms and treatment. Circulation. 2002;105(12):1503-8.

2. Sabbah HN, Sharov VG, Lesch M, Goldstein S. Progression of heart failure: a role for interstitial fibrosis. Mol Cell Biochem. 1995;147(1-2):29-34.

3. Varnava AM, Elliott PM, Sharma S, McKenna WJ, Davies MJ. Hypertrophic cardiomyopathy: the interrelation of disarray, fibrosis, and small vessel disease. Heart. 2000;84(5):476-82.

4. Shirani J, Pick R, Roberts WC, Maron BJ. Morphology and significance of the left ventricular collagen network in young patients with hypertrophic cardiomyopathy and sudden cardiac death. J Am Coll Cardiol. 2000;35(1):36-44.

5. Mewton N, Liu CY, Croisille P, Bluemke D, Lima JA. Assessment of myocardial fibrosis with cardiovascular magnetic resonance. J Am Coll Cardiol. 2011;57(8):891-903.

6. Kim RJ, Judd RM. Gadolinium-enhanced magnetic resonance imaging in hypertrophic cardiomyopathy: in vivo imaging of the pathologic substrate for premature cardiac death? J Am Coll Cardiol. 2003;41(9):1568-72.

7. Iles L, Pfluger H, Phrommintikul A, Cherayath J, Aksit P, Gupta SN, Kaye DM, Taylor AJ. Evaluation of diffuse myocardial fibrosis in heart failure with cardiac magnetic resonance contrast-enhanced T1 mapping. J Am Coll Cardiol. 2008;52(19):1574-80.

8. Flett AS, Hayward MP, Ashworth MT, Hansen MS, Taylor AM, Elliott PM, McGregor C, Moon JC. Equilibrium contrast cardiovascular magnetic resonance for the measurement of diffuse myocardial fibrosis: preliminary validation in humans. Circulation. 2010;122(2):138-44.

9. Chan W, Duffy SJ, White DA, Gao XM, Du XJ, Ellims AH, Dart AM, Taylor AJ. Acute left ventricular remodeling following myocardial infarction: coupling of regional healing with remote extracellular matrix expansion. JACC Cardiovasc Imaging. 2012;5(9):884-93.

10. Ling LH, Kistler PM, Ellims AH, Iles LM, Lee G, Hughes GL, Kalman JM, Kaye DM, Taylor AJ. Diffuse Ventricular fibrosis in atrial fibrillation: noninvasive evaluation and relationships with aging and systolic dysfunction. J Am Coll Cardiol. 2012;60:2402-8.

11. Ellims AH, lles LM, Ling LH, Hare JL, Kaye DM, Taylor AJ. Diffuse myocardial fibrosis in hypertrophic cardiomyopathy can be identified by cardiovascular magnetic resonance, and is associated with left ventricular diastolic dysfunction. J Cardiovasc Magn Reson. 2012;14(1):76.

12. Mascherbauer J, Marzluf BA, Tufaro C, Pfaffenberger S, Graf A, Wexberg P, Panzenbock A, Jakowitsch J, Bangert C, Laimer D, et al. Cardiac magnetic resonance postcontrast $\mathrm{T} 1$ time is associated with outcome in patients with heart failure and preserved ejection fraction. Circ Cardiovasc Imaging. 2013;6(6):1056-65.

13. Sibley CT, Noureldin RA, Gai N, Nacif MS, Liu S, Turkbey EB, Mudd JO, van der Geest RJ, Lima JA, Halushka MK, et al. T1 mapping in cardiomyopathy at cardiac MR: comparison with endomyocardial biopsy. Radiology. 2012;265(3):724-32.

14. Vickers KC, Rye KA, Tabet F. MicroRNAs in the onset and development of cardiovascular disease. Clin Sci Lond. 2014;126(3):183-94.

15. Jiang $X$, Tsitsiou E, Herrick SE, Lindsay MA. MicroRNAs and the regulation of fibrosis. FEBS J. 2010;277(9):2015-21.

16. Vettori S, Gay S, Distler O. Role of MicroRNAs in fibrosis. Open Rheumatol J. 2012;6:130-9.

17. Chim SS, Shing TK, Hung EC, Leung TY, Lau TK, Chiu RW, Lo YM. Detection and characterization of placental microRNAs in maternal plasma. Clin Chem. 2008:54(3):482-90

18. Creemers EE, Tijsen AJ, Pinto YM. Circulating MicroRNAs: novel biomarkers and extracellular communicators in cardiovascular disease? Circ Res. 2012;110(3):483-95

19. Fichtlscherer S, Zeiher AM, Dimmeler S. Circulating microRNAs: biomarkers or mediators of cardiovascular diseases? Arterioscler Thromb Vasc Biol. 2011;31(11):2383-90.

20. Maron BJ, McKenna WJ, Danielson GK, Kappenberger LJ, Kuhn HJ, Seidman CE, Shah PM, Spencer WH 3rd, Spirito P, Ten Cate FJ. American College of Cardiology/European Society of Cardiology clinical expert consensus document on hypertrophic cardiomyopathy. A report of the American College of Cardiology Foundation Task Force on Clinical Expert Consensus Documents and the European Society of Cardiology Committee for Practice Guidelines. J Am Coll Cardiol. 2003:42(9):1687-713.
21. Fang $L$, Beale $A$, Ellims AH, Moore XL, Ling LH, Taylor AJ, Chin-Dusting J, Dart AM. Associations between fibrocytes and postcontrast myocardial T1 times in hypertrophic cardiomyopathy. J Am Heart Assoc. 2013;2(5):e000270.

22. Gai N, Turkbey EB, Nazarian S, van der Geest RJ, Liu CY, Lima JA, Bluemke DA. T1 mapping of the gadolinium-enhanced myocardium: adjustment for factors affecting interpatient comparison. Magn Reson Med. 2011;65(5):1407-15.

23. Querejeta R, Varo N, Lopez B, Larman M, Artinano E, Etayo JC, Martinez Ubago JL, Gutierrez-Stampa M, Emparanza Jl, Gil MJ, et al. Serum carboxyterminal propeptide of procollagen type I is a marker of myocardial fibrosis in hypertensive heart disease. Circulation. 2000;101(14):1729-35.

24. Velagaleti RS, Gona P, Sundstrom J, Larson MG, Siwik D, Colucci WS, Benjamin EJ, Vasan RS. Relations of biomarkers of extracellular matrix remodeling to incident cardiovascular events and mortality. Arterioscler Thromb Vasc Biol. 2010;30(11):2283-8.

25. Kupari M, Laine M, Turto H, Lommi J, Werkkala K. Circulating collagen metabolites, myocardial fibrosis and heart failure in aortic valve stenosis. J Heart Valve Dis. 2013;22(2):166-76.

26. Roncarati R, Viviani Anselmi C, Losi MA, Papa L, Cavarretta E, Da Costa Martins P, Contaldi C, Saccani Jotti G, Franzone A, Galastri L, et al. Circulating miR-29a, among other up-regulated microRNAs, is the only biomarker for both hypertrophy and fibrosis in patients with hypertrophic cardiomyopathy. J Am Coll Cardiol. 2014;63(9):920-7.

27. Ellims AH, Shaw JA, Stub D, Iles LM, Hare JL, Slavin GS, Kaye DM, Taylor AJ. Diffuse myocardial fibrosis evaluated by post-contrast 11 mapping correlates with left ventricular stiffness. J Am Coll Cardiol. 2014;63(11):1112-8.

28. Thum T, Gross C, Fiedler J, Fischer T, Kissler S, Bussen M, Galuppo P, Just S, Rottbauer W, Frantz S, et al. MicroRNA-21 contributes to myocardial disease by stimulating MAP kinase signalling in fibroblasts. Nature. 2008:456(7224):980-4

29. van Rooij E, Sutherland LB, Thatcher JE, DiMaio JM, Naseem RH, Marshall WS, Hill JA, Olson EN. Dysregulation of microRNAs after myocardial infarction reveals a role of miR-29 in cardiac fibrosis. Proc Natl Acad Sci USA. 2008;105(35):13027-32.

30. Duisters RF, Tijsen AJ, Schroen B, Leenders JJ, Lentink V, van der Made I, Herias V, van Leeuwen RE, Schellings MW, Barenbrug P, et al. miR-133 and miR-30 regulate connective tissue growth factor: implications for a role of microRNAs in myocardial matrix remodeling. Circ Res. 2009;104(2):170-8

\section{(176p following 178)}

31. van Almen GC, Verhesen W, van Leeuwen RE, van de Vrie M, Eurlings $C$, Schellings MW, Swinnen M, Cleutjens JP, van Zandvoort MA, Heymans S, et al. MicroRNA-18 and microRNA-19 regulate CTGF and TSP-1 expression in age-related heart failure. Aging Cell. 2011;10(5):769-79.

32. Wang B, Koh P, Winbanks C, Coughlan MT, McClelland A, Watson A, Jandeleit-Dahm K, Burns WC, Thomas MC, Cooper ME, et al. miR-200a Prevents renal fibrogenesis through repression of TGF-beta2 expression. Diabetes. 2011;60(1):280-7.

33. Chung AC, Huang XR, Meng X, Lan HY. miR-192 mediates TGF-beta/ Smad3-driven renal fibrosis. J Am Soc Nephrol. 2010;21(8):1317-25.

34. Han X, Yan S, Weijie Z, Feng W, Liuxing W, Mengquan L, Qingxia F. Critical role of miR-10b in transforming growth factor-beta1-induced epithelial-mesenchymal transition in breast cancer. Cancer Gene Ther. 2014;21(2):60-7.

35. Kato $M$, Zhang J, Wang M, Lanting L, Yuan H, Rossi JJ, Natarajan R. MicroRNA-192 in diabetic kidney glomeruli and its function in TGF-betainduced collagen expression via inhibition of E-box repressors. Proc Natl Acad Sci USA. 2007;104(9):3432-7.

36. Sheedy FJ, O'Neill LA. Adding fuel to fire: microRNAs as a new class of mediators of inflammation. Ann Rheum Dis. 2008;67(Suppl 3):iii50-5.

37. Taganov KD, Boldin MP, Chang KJ, Baltimore D. NF-kappaB-dependent induction of microRNA miR-146, an inhibitor targeted to signaling proteins of innate immune responses. Proc Natl Acad Sci USA. 2006;103(33):12481-6.

38. Guo CJ, Pan Q, Li DG, Sun H, Liu BW. miR-15b and miR-16 are implicated in activation of the rat hepatic stellate cell: an essential role for apoptosis. J Hepatol. 2009;50(4):766-78.

39. Wurdinger T, Tannous BA, Saydam O, Skog J, Grau S, Soutschek J, Weissleder R, Breakefield XO, Krichevsky AM. miR-296 regulates growth factor receptor overexpression in angiogenic endothelial cells. Cancer Cell. 2008;14(5):382-93. 
40. Tijsen AJ, Pinto YM, Creemers EE. Non-cardiomyocyte microRNAs in heart failure. Cardiovasc Res. 2012;93(4):573-82.

41. McCall MN, Kent OA, Yu J, Fox-Talbot K, Zaiman AL, Halushka MK. MicroRNA profiling of diverse endothelial cell types. BMC Med Genomics. 2011:4:78

42. Feng B, Chen S, McArthur K, Wu Y, Sen S, Ding Q, Feldman RD, Chakrabarti S. miR-146a-Mediated extracellular matrix protein production in chronic diabetes complications. Diabetes. 2011;60(11):2975-84.
43. Hecker M, Thamilarasan M, Koczan D, Schroder I, Flechtner K, Freiesleben S, Fullen G, Thiesen HJ, Zettl UK. MicroRNA expression changes during interferon-beta treatment in the peripheral blood of multiple sclerosis patients. Int J Mol Sci. 2013;14(8):16087-110.

44. Valadi H, Ekstrom K, Bossios A, Sjostrand M, Lee JJ, Lotvall JO. Exosomemediated transfer of mRNAs and microRNAs is a novel mechanism of genetic exchange between cells. Nat Cell Biol. 2007;9(6):654-9.
Submit your next manuscript to BioMed Central and take full advantage of:

- Convenient online submission

- Thorough peer review

- No space constraints or color figure charges

- Immediate publication on acceptance

- Inclusion in PubMed, CAS, Scopus and Google Scholar

- Research which is freely available for redistribution

Submit your manuscript at

www.biomedcentral.com/submit

() BioMed Central 